\title{
PROBABLE VARIABILITY OF NGC 6572
}

\author{
D. KOELBLOED \\ (Astronomical Institute of the University of Amsterdam, The Netherlands)
}

\section{Introduction}

The suspected rapid rate of evolution of the central stars of planetary nebulae (Shklovsky, 1956) and the discovery by Seaton (1966) of their evolutionary track in the $\mathrm{H}-\mathrm{R}$ diagram, have led to renewed interest in these stars. According to Seaton the evolutionary track starts at an atmospheric temperature of about $30000^{\circ} \mathrm{K}$ and a luminosity of $100 L_{\odot}$. A maximum brightness is reached of $25000 L_{\odot}$ at $T_{*}=70000^{\circ} \mathrm{K}$. Then $T_{*}$ increases to $100000^{\circ} \mathrm{K}$, at approximately constant $L$, whereafter, at this temperature, $L$ decreases finally below $100 L_{\odot}$. This whole evolution is believed to take place in only $5 \times 10^{4}$ years. At such a rapid evolution, short-period fluctuations in brightness are expected and it should even be possible to detect secular variations in a lifetime. Aller and Liller (1957) discovered the variability of the spectrum of IC 4997 and Vorontsov-Velyaminov (1961) found variations in line intensities of NGC 6905. Khromov (1962) tried to explain the variations in both nebulae as a result of temperature changes of the central stars. Variations in the emission spectrum of IC 4997 were explained by Aller and Liller (1966) as a result of nebular expansion.

Changes, since the beginning of this century, of diameters of planetary nebulae were studied by Martha Liller et al. (1966) and of the magnitudes of central stars by Kohoutek (1966). In this note we compare $A_{v}$-values of NGC 6572 , derived in the interval 1927-66.

\section{Comparison of $A_{v}$-Values of NGC 6572 (1927-66)}

\section{A. ZANSTRA'S MEASUREMENTS (1927-46)}

Just 40 years ago (September 1927) Zanstra $(1930,1931)$ photographed the first standardized slitless spectra of NGC 6572 and NGC 6543 in order to derive the temperatures of the central stars, with his well-known $A_{v}$-method. With this method one compares the total intensity of a monochromatic nebular image of an element with the intensity of the continuum of the central star at the same wavelength. The superiority, compared with other methods, is that only relative measurements are used, which need no interstellar-reddening corrections. When old and new observations are

Osterbrock and O'Dell (eds.), Planetary Nebulae, 376-380. 6.A.U. 
compared a change in response characteristics in the meantime has no effect on the results, an especially important consideration.

The author noticed that Zanstra's new determinations of the temperature-parameters $T_{\mathrm{H}}$ (Zanstra, 1960 ) $52000^{\circ} \mathrm{K}$ in 1938 and $54000^{\circ} \mathrm{K}$ in 1946 , were considerably higher than the older ones, $41000^{\circ}$, found by him in 1927 (Zanstra, 1930, 1931). The old low values were confirmed by Berman (1930). Berman found $T_{\mathrm{H}}=43500^{\circ} \mathrm{K}$ from photographs taken by him in 1928 .

For detecting variability, we cannot compare the published temperatures, because they strongly depend on the method used.* We have to compare the original $A_{v}$-values. They are collected in Table 1 . Berman used only $H \delta$, but did not publish the $A_{v^{-}}$ value.

Table 1

\section{Comparison of $A_{v}$-values of NGC 6572}

1927

(1)

$\mathbf{H} \beta$
$\mathbf{H} \gamma$
$\mathbf{H} \delta$
$\mathrm{HeI}, \lambda 4471$
$\mathrm{HeI}, \lambda 4026$

(3)

0.20

0.075

0.039

0.009
1938-46

(4)

$(0 \cdot 118)$

$(0.070)$

0.019

0.0066
1966

$0 \cdot 265$

0.128

(0.110)

0.067

0.0147

Column (2) are the values published by Zanstra (1930).

Column (3) are improved $A_{v}$-values derived from the same negatives and published by Zanstra (1931).

As on the plates of 1938 and 1946, taken by Aller, the Balmer images were too strong to give reliable results, Zanstra had to use the $A_{v}$-values of the HeI lines $4471 \AA$ and $4026 \AA$ to find those for hydrogen. He therefore had to rely on the nebular lineintensity ratios $\mathrm{H} \delta / 4026=10 \cdot 5$ and $\mathrm{H} \gamma / 4471=6 \cdot 2$ found in the literature (cf. Bull. astr. Inst. Netherl., 15, 244).

In column (4) the 1938-46 results are given. The values in parenthesis were derived from the $\mathrm{He}$ lines as described above.

Comparison of columns (2) and (3) with (4) shows that an increase of the $A_{v}$-values with a factor of about 2 seems indicated. As it is improbable that such a large factor can be explained by observational errors one has to consider the possibility of a temperature increase of the central star in the short interval 1927-1938.

A systematic error between the old and new series is contradicted by the comparison

* For NGC 6572 see also the temperatures derived by Seaton in Report Prog. Physics, 23, 1961, 313, and Mon. Not. R. astr. Soc., 132, 1966, 29. 
of the results for NGC 6543 where only $A(\mathrm{H} \delta)$ can be compared. We find $A(\mathrm{H} \delta)=$ $0.039 \pm 0.006$ (1927) from two films and $0.032 \pm 0.004$ (1938-46) from three plates.

\section{B. ALLER'S PHOTOELECTRIC TRACINGS ( 1966 )}

In connection with the suspected variability it seemed worthwhile to have new observations. At the request of the author Dr. Lawrence H. Aller, in cooperation with G.J. Stanley and S. J. Czyzak, secured observations of NGC 6572, on May 22, 1966, with the photoelectric scanner on the 60 -inch Mount Wilson reflector. The tracings were kindly put at the author's disposal.

The continuum, on the tracings, contains contributions of both the star and the nebula. On spectra kindly loaned by Dr. Olin C. Wilson, no nebular continuum could be detected; also, Page (1942) was unable to measure it on plates, taken in 1939 and 1940, especially for the study of the nebular continuum. Neglecting this continuum, we find the $A_{v}$-values given in column (5). For $\mathrm{H} \gamma$ two values are given. The photoelectric profiles of $\mathrm{H} \beta, \mathrm{H} \delta$ and $\mathrm{He}$ I 4471 are symmetric whereas the $\mathrm{H} \gamma$ profile is disturbed at the red wing. Adopting a symmetrical profile for $\mathrm{H} \gamma$ too, we find the value $0 \cdot 128$. The disturbance is caused by the $\mathrm{Hg}$ line $4359 \AA$ of the sky background and by [O III] $4363 \AA$ of the nebular spectrum. According to Aller and Kaler (1964) the ratio to the nebular lines $\mathrm{H} \gamma / 4363$ is $4 \cdot 3$, derived from observations made in 1959-63. Correcting the asymmetrical line for both lines we find the value in parenthesis. The values for $\mathrm{H} \beta$ have low weight. On the photographic plates $\mathrm{H} \beta$ is disturbed by the strong N2nebulium line, whereas on the photoelectric tracing the stellar continuum is too faint for deriving good results.

\section{Conclusions}

We conclude that the photoelectric results are in good agreement with Zanstra's 1938-46 results. Thus, if the change is real, it appeared to have occurred chiefly between 1927 and 1938. The results point out the desirability of further observations at regular intervals of planetary nebulae.

\section{Further Evidences of Variability}

In addition, two independent indications of variability of NGC 6572 can be mentioned. Strong evidence of an increase of the nebular diameter was given by Martha Liller et al. (1965). They compared photographs taken in 1916 and 1961 with the Crossley-reflector. Of the 14 nebulae investigated, the smallest probable error in the nebular growth was found for the minor axis of NGC 6572. The mean increase in angular size is 0 " $81 \pm 0 \cdot 10$ in 100 years, based on a continuous increase during 1916-61. This corresponds to an increase of the mean diameter of a factor of 1.07 if the image of 1916 is compared with that of 1961 . 
The second indication of variability is the determination of the photographic magnitude of the central star. Kohoutek (1966) measured an AG K2 plate (1929) and an AG K3 plate (1961) and found an increase in luminosity of 0.3 . He states that this difference is too large to be accounted for by observational errors. An increase of $0 .{ }^{\mathrm{m}} 3$ in the photographic region corresponds roughly to an increase from $T_{*}=43000^{\circ} \mathrm{K}$ to $53000^{\circ} \mathrm{K}$, if $r_{*}$ remains constant.

The variations of $A_{v}$, the diameter and $m_{\mathrm{pg}}$ may be explained by an increase of the temperature of the central star with a corresponding increase of the diameter of the surrounding HiI region.

\section{The Temperature Parameters $T_{\mathrm{H}}$ and $T_{\mathrm{Hel}}$ of NGC 6572}

From the presence of [OI] lines in the nebular spectrum, it follows that the absorption by $\mathrm{HI}$ is complete in NGC 6572. Assuming with Zanstra an electron temperature of $15000^{\circ} \mathrm{K}$, we find, using Zanstra's method (1960), $T_{\mathrm{H}}=53300^{\circ} \mathrm{K}$ from the $1966 \mathrm{~A}$ $(\mathrm{H} \delta)$-value. The same temperature is found from the $A(\mathrm{H} \gamma)$ (symmetrical profile) and from the $A(\mathrm{He}, 4471 \AA)$. The $A(\mathrm{H} \gamma)$ (corrected asymmetrical profile) gives $50800^{\circ} \mathrm{K}$. As the $A(\mathrm{H} \delta)$ has the larger weight we adopt $T_{\mathrm{H}}=53300^{\circ} \mathrm{K}$.

In Figure $1 T_{\mathrm{He}}$ is plotted against $T_{\mathrm{H}}$ for Zanstra's group I (1960) (high-accuracy measurements). The 1966 value for NGC 6572 is added (black dot).

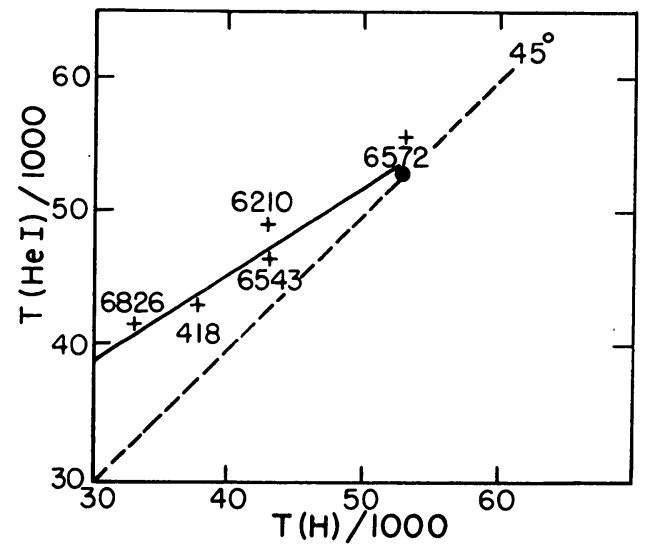

FIG. 1. The He I Zanstra temperature plotted against the Hydrogen Zanstra temperature. The dashed line shows the one to one relation.

According to Hummer and Seaton (1963) the helium images can only be used for temperature determinations if $T_{*}<50000^{\circ} \mathrm{K}$. All nebulae of group I, with NGC 6572 as a probable boundary case, satisfy the condition.

An increase of the difference between the two temperatures for lower temperatures seems indicated. 
From the approximate equality of $T_{\mathrm{H}}$ and $T_{e e}$ (derived from forbidden lines) Zanstra concludes complete absorption by hydrogen for group I. Then the explanation of Figure 1 - if confirmed by more observations - must follow from atmospheric models of the central star (Gebbie and Seaton, 1963; Böhm and Deinzer, 1965a, b; Gebbie, 1967).

Seaton (1966, table I) on the other hand, arranges NGC 6572 and 6543 in class $\mathrm{a}_{1}$ (thick HeII, thick $\mathrm{HI}_{\mathrm{I}}$ ) and NGC 6826 in class $\mathrm{a}_{2}$ (thick He II, thin $\mathrm{HI}$ ). In this case the completeness of absorption in the nebulae has to be considered too in discussing Figure 1.

\section{Acknowledgements}

The author is very thankful to Dr. L.H. Aller and his collaborators for making the photoelectric scans of NGC 6572.

\section{References}

Aller, L.H., Kaler, J.B. (1964) Astrophys. J., 140, 621.

Aller, L. H., Liller, W. (1957) Sky and Telescope, 161, 222.

Aller, L.H., Liller, W. (1966) Mon. Not. R. astr. Soc., 132, 337.

Aller, L.H., Zanstra, H. (1960) Bull. astr. Inst. Netherl., 15, 249.

Berman, L. (1930) Lick Obs. Bull., 430.

Böhm, K.H., Deinzer, W. (1965a) Z. Astrophys., 61, 1.

Böhm, K. H., Deinzer, W. (1965b) Z. Astrophys., 63, 177.

Gebbie, K. B., Seaton, M.J. (1963) Nature, 199, 580.

Gebbie, K. B. (1967) Mon. Not. R. astr. Soc., 135, 181.

Harman, R.J., Seaton, M.J. (1966) Mon. Not. R. astr. Soc., 132, 15.

Hummer, D.G., Seaton, M.J. (1963) Mon. Not. R. astr. Soc., 125, 437.

Hummer, D. (1964) Mon. Not. R. astr. Soc., 127, 240.

Kohoutek, L. (1966) Bull. astr. Inst. Csl., 17, 318.

Khromov, G.S. (1962) Sov. Astr. - A. J., 5, 619.

Liller, Martha H., Welther, Barbara L., Liller, W. (1966) Astrophys. J., 144, 280.

Page, T. (1942) Astrophys. J., 96, 78.

Seaton, M.J. (1966) Mon. Not. R. astr. Soc., 132, 113.

Shklovsky, I.S. (1956) Russian A. J., 33, 315.

Vorontsov-Velyaminov, B. (1961) Astr. Zu., 38, 247. (Also in Soviet Astr. - A. J., 5, 186.)

Zanstra, H. (1930) Publ. Dom. astrophys. Obs., Victoria, 4, 209.

Zanstra, H. (1931) Z. Astrophys., 2, 1.

Zanstra, H. (1960) Bull. astr. Inst. Netherl., 15, 237. 